\title{
BMJ Open Statin and dual antiplatelet therapy for the prevention of early neurological deterioration and recurrent stroke in branch atheromatous disease: a protocol for a prospective single-arm study using a historical control for comparison
}

\author{
Yen-Chu Huang (D) , , Jiann-Der Lee, ${ }^{1,2}$ Hsu-Huei Weng (D) , ${ }^{3}$ Leng-Chieh Lin, ${ }^{4}$ \\ Yuan-Hsiung Tsai (i) ,2,5 Jen-Tsung Yang ${ }^{2,6}$
}

To cite: Huang Y-C, Lee J-D, Weng $\mathrm{H}-\mathrm{H}$, et al. Statin and dual antiplatelet therapy for the prevention of early neurological deterioration and recurrent stroke in branch atheromatous disease: a protocol for a prospective single-arm study using a historical control for comparison. BMJ Open 2021;11:e054381. doi:10.1136/ bmjopen-2021-054381

- Prepublication history for this paper is available online. To view these files, please visit the journal online (http://dx.doi org/10.1136/bmjopen-2021054381).

Received 12 June 2021 Accepted 01 November 2021

Check for updates

(c) Author(s) (or their employer(s)) 2021. Re-use permitted under CC BY-NC. No commercial re-use. See rights and permissions. Published by BMJ.

For numbered affiliations see end of article.

Correspondence to Dr Jen-Tsung Yang; jents716@ms32.hinet.net

\section{ABSTRACT}

Introduction Branch atheromatous disease (BAD)

contributes to small-vessel occlusion in cases of

occlusion or stenosis of large calibre penetrating arteries, and it is associated with a higher possibility of early neurological deterioration (END) and recurrent stroke in acute ischaemic stroke. As the pathology of BAD is due to atherosclerosis, we postulate that early intensive medical treatment with dual antiplatelet therapy (DAPT) and highintensity statins may prevent END and recurrent stroke in acute small subcortical infarction caused by BAD.

Methods and analysis In this prospective, singlecentre, open-label, non-randomised, single-arm study using a historical control, we will compare early DAPT and high-intensity statin treatment with a historical control group of patients with BAD who were treated with single antiplatelet therapy without high-intensity statin treatment. Patients will be eligible for enrolment if they are admitted for acute ischaemic stroke within 24 hours, have a National Institutes of Health Stroke Scale (NIHSS) score of $1-8$ and are diagnosed with BAD by MRI. Patients will take aspirin, clopidogrel and high-intensity statins (atorvastatin or rosuvastatin) within 24 hours of stroke onset, followed by aspirin or clopidogrel alone from day 22. The primary endpoint is the percentage of patients who develop END within 7 days of stroke onset (defined as an increase in the NIHSS score $\geq 2$ points) and recurrent stroke within 30 days. The total sample sizes will be 138 for the intervention group and 277 for the control group. A historical control group will be drawn from previous prospective observation studies.

Ethics and dissemination The protocol of this study has been approved by the Institutional Review Board of Chang Gung Memorial Hospital (202001386A3). All participants will have to sign and date an informed consent form. The findings arising from this study will be disseminated in peer-reviewed journals and academic conferences.

Trial registration number NCT04824911.
Strengths and limitations of this study

- This will be the first trial to evaluate the effectiveness of dual antiplatelet therapy (DAPT) and high-intensity statins for the prevention of early neurological deterioration and recurrent stroke in patients with acute ischaemic stroke from branch atheromatous disease.

- This trial will recruit patients with National Institutes of Health Stroke Scale scores of 1-8, which are more severe than current guideline suggestion of scores $\leq 3$ for mild stroke with DAPT.

- A randomised controlled study with a parallel control arm receiving single antiplatelet treatment for mild stroke is against the latest guidelines, so it is inevitable to conduct this trial with a historical control group.

- The use of a historical control group has the inherent drawbacks of non-randomisation and non-blinding, so we cannot exclude the possibility of selection, performance, detection and attrition bias.

- Because DAPT and high-intensity statin treatment are administered simultaneously, it is unable to know each treatment effect.

\section{INTRODUCTION}

Small-vessel occlusion is the most common stroke subtype in Asians; it is caused by the occlusion of deep perforating arteries, mainly by the underlying pathologies of lipohyalinosis or cerebral amyloid angiopathy. ${ }^{12}$ Branch atheromatous disease (BAD) has also been reported to contribute to small-vessel occlusion in cases of occlusion or stenosis that occur at the origin of large calibre penetrating arteries, due to microatheromas or junctional atherosclerotic plaques. ${ }^{3} \mathrm{BAD}$ is associated with an increased possibility of early neurological deterioration (END) and 
recurrent stroke, especially progressive motor deficits, compared with infarction due to hypertensive arteriopathy. ${ }^{4-6}$ It has been proposed that BAD should be classified as large artery atherosclerosis rather than small vessel occlusion.?

Following acute ischaemic stroke, single antiplatelet agents, such as aspirin or clopidogrel, are the mainstay treatment for recurrent stroke prevention. However, the modest effects of single antiplatelet therapy led to the study of combination antiplatelet therapy for the prevention of recurrent stroke. The Clopidogrel in High-Risk Patients with Acute Nondisabling Cerebrovascular Events and the Platelet-Oriented Inhibition in New TIA and Minor Ischemic Stroke trials showed that dual antiplatelet therapy (DAPT) might be beneficial for reducing the probability of ischaemic events in patients with high-risk transient ischaemic attack (TIA) or minor stroke. ${ }^{89}$ However, these studies included all non-embolic ischaemic stroke types. Some studies also showed that DAPT reduced the possibility of END. ${ }^{10}{ }^{11}$ A retrospective observation study showed that DAPT was beneficial for the primary outcomes of stroke recurrence, myocardial infarction and all-cause death in patients with large atherosclerotic stroke. ${ }^{12}$

For patients with acute ischaemic stroke, the guidelines suggest starting or continuing statin treatment as soon as oral medications can be used safely. ${ }^{13}$ Current guidelines also recommend high-intensity or moderate-intensity statin therapy, independent of the baseline low-density lipoprotein cholesterol (LDL-C), to reduce the risk of stroke and cardiovascular events for patients with TIA or ischaemic stroke of atherosclerotic origin. ${ }^{14}$ High-dose statin treatment in the acute phase of ischaemic stroke and TIA significantly reduced National Institutes of Health Stroke Scale (NIHSS) scores and improved shortterm functional outcomes without increasing related adverse events ${ }^{15}$; it also effectively stabilised symptomatic intracranial atherosclerotic plaques as documented by high-resolution MRI. ${ }^{16}$

DAPT and high-intensity statins are the mainstay treatments for intracranial atherosclerosis (ICAS).${ }^{17}$ As BAD and ICAS share the same pathology as atherosclerosis, we postulate that early intensive medical treatment with DAPT and high-intensity statins may prevent END and recurrent stroke. This study aims to evaluate whether intensive medical therapy with aspirin, clopidogrel and high-intensity statins can prevent END and recurrent stroke in acute small subcortical infarction caused by BAD.

\section{METHODS}

\section{Study design}

In this prospective, single-centre, open-label, nonrandomised, single-arm, historically controlled study, we will compare early intensive treatment for BAD initiated within 24 hours of stroke onset, using DAPT (aspirin plus clopidogrel) and high-intensity statin treatment, with a historical control group of patients with BAD treated with single antiplatelet therapy, without high-intensity statin treatment. $^{18} 19$ The study will be conducted in Chang Gung Memorial Hospital at Chiayi, Taiwan, from March 2021 to February 2023.

\section{Study population}

Patients are eligible to participate if they meet the following inclusion criteria: (1) have a clinical diagnosis of ischaemic stroke with an NIHSS score of 1-8, (2) have an ischaemic lesion on diffuse-weighted imaging (DWI) located in the striatocapsular territory or brain stem areas, with an axial diameter of $\leq 20 \mathrm{~mm}$, (3) have $\mathrm{BAD}$, defined by a visible ischaemic lesion in three or more axial cuts on DWI in the lenticulostriate territory or infarcts that extend from the basal surface of the pons and (4) could receive intensive medical treatment within 24 hours of stroke onset. Patients with $>50 \%$ stenosis of the relevant arteries on magnetic resonance angiography (MRA), including intracranial or extracranial internal carotid artery, middle cerebral artery or basilar artery, will be excluded. We will also exclude patients at high risk of cardioembolic stroke, such as those with atrial fibrillation or heart failure. Full details of the inclusion and exclusion criteria are listed in Box 1.

A historical control group will be drawn from our prospective observation studies, which have been executed between January 2011 and December 2020, and aimed to evaluate and predict END or atrial fibrillation. ${ }^{18}{ }^{19}$ Patients will be selected if they fulfil the following inclusion criteria: took aspirin or clopidogrel alone and did not receive high-intensity statin treatment. Patients in the historical control group will have received statin treatment once their total cholesterol was $\geq 160 \mathrm{mg} / \mathrm{dL}$ or their LDL-C was $\geq 100 \mathrm{mg} / \mathrm{dL}$. High-intensity statin treatment includes atorvastatin $40-80 \mathrm{mg}$ or rosuvastatin 20-40 mg daily. ${ }^{20}$ All clinical information and outcomes have been prospectively recorded.

\section{Trial intervention}

Dual antiplatelet and high-intensity statin treatment will be administered within 24 hours of stroke onset (figure 1). Dual antiplatelet treatment includes aspirin (300 mg loading and $100 \mathrm{mg} /$ day) and clopidogrel (300 mg loading and $75 \mathrm{mg} /$ day) and high-intensity statins includes atorvastatin $40-80 \mathrm{mg} /$ day or rosuvastatin $20 \mathrm{mg} /$ day. Patients will take aspirin and clopidogrel for 21 days and then just take aspirin or clopidogrel alone. High-intensity statins will be maintained for 90 days, but a decreased dose is allowed if any side effects occur, including elevated liver functions, elevated creatine kinase and myalgia or at the judgement of the treating physician. We will follow-up with alanine aminotransferase (ALT) and creatine kinase 14 days after treatment and will follow-up with ALT, creatine kinase and LDL-C 90 days after treatment. Patients with atherosclerotic plaques in the first MRI will keep high-intensity statin treatment for 6 months. 
Box 1 Inclusion and exclusion criteria (issue date: 1

September 2020)

\section{Inclusion criteria:}

- Clinical diagnosis of ischaemic stroke with a National Institute of Health Stroke Scale (NIHSS) score of 1-8.

- An ischaemic lesion on diffuse-weighted imaging (DWI) located in the striatocapsular territory or brain stem areas, with an axial diameter of $\leq 20 \mathrm{~mm}$.

- Branch atheromatous disease, defined by a visible ischaemic lesion in three or more axial cuts on DWI in the lenticulostriate territory or infarcts that extend from the basal surface of the pons.

- Ability to participate within 24 hours of the time of last known free of new ischaemic symptoms.

- MRI of the head ruling out haemorrhage or other pathologies, such as vascular malformation, tumour or abscess, that could explain their symptoms or contraindicate therapy.

- Ability to tolerate high-intensity medical therapy, including aspirin at a dose of $50-325 \mathrm{mg} /$ day, clopidogrel at $300 \mathrm{mg}$ loading and $75 \mathrm{mg}$ after day 2 and high-intensity statins (either atorvastatin $40-80 \mathrm{mg} /$ day or rosuvastatin $20 \mathrm{mg} /$ day).

- Pre-stroke modified Rankin Scale score of $\leq 1$.

Exclusion criteria:

- Age $<18$ years.

- At the judgement of the treating physician.

- A candidate for thrombolysis, endarterectomy or endovascular intervention.

- Receipt of any intravenous or intra-arterial thrombolysis within 1 week prior to the index event.

- Patients with $>50 \%$ stenosis of the relevant arteries as determined by magnetic resonance angiography, including the intracranial or extracranial internal carotid artery, middle cerebral artery or basilar artery.

- Patients with a high risk of cardioembolic stroke, such as those with atrial fibrillation, acute myocardial infarction, severe heart failure or valvular heart disease.

- Those with other determined stroke aetiologies, such as vasculitis, shock, antiphospholipid antibody syndrome, etc.

- Gastrointestinal bleeding or major surgery within 3 months prior to the index event.

- History of non-traumatic intracranial haemorrhage.

- Clear indication for anticoagulation during the study period (deep venous thrombosis, pulmonary embolism or hypercoagulable state).

- Qualifying ischaemic event induced by angiography or surgery.

- Severe non-cardiovascular comorbidity with a life expectancy $<3$ months.

- Contraindication to clopidogrel, aspirin, atorvastatin or rosuvastatin.

- Known allergy to clopidogrel, aspirin atorvastatin or rosuvastatin.

- Severe renal (serum creatinine: $>2 \mathrm{mg} / \mathrm{dL}$ ) or hepatic insufficiency (International normalized ratio: $>1.2 ;$ ALT: $>40 \mathrm{U} / \mathrm{L}$ or any resultant complication, such as variceal bleeding, encephalopathy or jaundice).

- Haemostatic disorder or systemic bleeding in the past 3 months.

- Current thrombocytopenia (platelet count: $<100 \times 10^{9} / \mathrm{L}$ ) or leucopenia $\left(<2 \times 10^{9} / \mathrm{L}\right)$.

- History of drug-induced haematologic or hepatic abnormalities.

- Anticipated requirement for long-term (>7 day) non-study antiplatelet drugs (eg, dipyridamole, ticagrelor and ticlopidine) or nonsteroidal anti-inflammatory drugs.

- Not willing or able to discontinue prohibited concomitant medications.

\section{Box 1 Continued}

- Women of childbearing age not practicing reliable contraception who do not have a documented negative pregnancy test.

- Other neurological conditions that would complicate assessment of outcomes during follow-up.

- Ongoing treatment in another study of an investigational therapy, or treatment in such a study within the last 7 days.

Neurological deficits will be evaluated by a stroke study nurse using the NIHSS, on day 1 , day 2 , day 3 , day 7 and day 90. As END in lacunar infarction is mainly associated with motor deficits, END is defined as an NIHSS score increase $\geq 2$ within 7 days of stroke onset. ${ }^{21}$ Clinical outcomes at admission and at 90 days will be evaluated using the modified Rankin Scale (mRS). A good outcome is defined as an mRS score $\leq 1$. Mortality at 3 months and any haemorrhagic complications will also be recorded. All examinations will be performed after obtaining written informed consent from the patients or the appropriate family members.

Patients with visible atheromatous plaques in the parental artery in the initial MRI will receive a follow-up MRI 6 months later to see the interval changes in atheromatous plaques.

\section{Study outcomes}

The primary outcome will be the percentage of patients with END within 7 days and recurrent ischaemic stroke within 30 days. Secondary outcomes will include: (1) the percentage of patients with favourable functional recovery, defined as an mRS $\leq 1$ on day 90 , (2) the percentage of patients with new clinical vascular events within 90 days, including ischaemic stroke, haemorrhagic stroke, TIA, myocardial infarction and vascular death, (3) changes in atherosclerotic

\section{Historical control group}

$$
\text { Aspirin or Clopidogrel alone }
$$

\section{Intervention group}

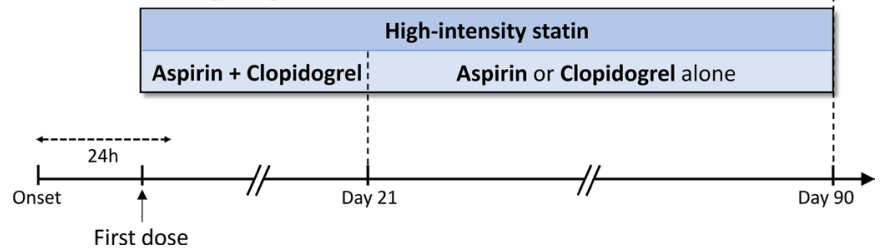

Figure 1 A schematic diagram of the treatment schedule and study design. In the intervention group, patients will take aspirin ( $300 \mathrm{mg}$ loading and $100 \mathrm{mg} /$ day), clopidogrel (300 mg loading and $75 \mathrm{mg} /$ day) and high-intensity statins (atorvastatin $40-80 \mathrm{mg} /$ day or rosuvastatin $20 \mathrm{mg} /$ day) within 24 hours of stroke onset, followed by aspirin or clopidogrel alone from day 22. High-intensity statins includes atorvastatin $40-80 \mathrm{mg} /$ day or rosuvastatin $20 \mathrm{mg} /$ day. In the historical control group, patients will be selected from previous prospective studies if they fulfil the following inclusion criteria: took aspirin or clopidogrel alone and did not receive high-intensity statin treatment. 
plaques, which will be measured by high-resolution MRI at the start of the study and after 6 months, (4) the number of moderate to severe bleeding events as defined by the Global Use of Strategies to Open Occluded Arteries (GUSTO) classification ${ }^{22}$ and (5) the total mortality rate.

\section{Sample size}

In single subcortical infarction, END was reported to occur frequently in $\mathrm{BAD}$ with an incidence of $27 \%$ in our previous cohort and $33.8 \%-40 \%$ in other studies. ${ }^{10} 23$ The END rate may decrease to $9.7 \%$ in patients with $\mathrm{BAD}$ who underwent combination antiplatelet therapy with cilostazol. ${ }^{10}$ The total sample sizes will be 138 for the intervention group and 277 for the control group. The estimated END rate is $27 \%$ for the control group and $15 \%$ for the intervention group, with $80 \%$ power and a two-sided $\alpha$ of 0.05 . The intent-totreat principle will be applied to the primary outcome analysis, and the sample size is, therefore, inflated to safeguard against 5\% lost to follow-up in the actual treatment groups, which could dilute the effect size.

\section{Statistical analysis}

Statistical analyses will be performed using the SPSS statistical software (V.25). The Kolmogorov-Smirnov test will be used to examine the normality of continuous variables. The Mann-Whitney U test and Student's t-test will be used to test for differences between the two groups, as appropriate. Categorical data will be analysed using the $\chi^{2}$ test. A propensity score matching analysis will be used to measure and balance predetermined covariates between two groups. A logistic regression model will be used to test independent variables for the measured outcomes. Variables showing a $\mathrm{p}$ value of $<0.1$ for univariate analysis will be entered into the multivariate logistic analysis using the forward selection method. All tests will be two-tailed, and a $p$ value of $<0.05$ is considered to indicate a statistically significant difference.

\section{Data management}

All the completed documents and the informed consent forms will be stored in a secured facility, under lock and key. The database for clinical data will be created using Access software and the access will be limited to principal investigators. A study steering committee will be established to ensure that the study was conducted to the required standards. The clinical research assistant will verify all consent forms, compliance with study protocol and procedures, and data quality. The research team will make half-yearly reports to the study steering committee. All the records and documents will be kept for 7 years after the completion of the study.

\section{Adverse events}

Any adverse events that occur during the conduct of the study will be reported to the Domain Specific Research Board, according to the local institutional policy. Interim analyses will be conducted by the study steering committee to monitor the accumulating data and to decide continuing or stopping the trial.

\section{Patient and public involvement}

Patients and members from stroke associations participated in the preparation and formulation of this proposal. Patients with acute ischaemic stroke from BAD will be involved in the trial. The associations will be involved in plans to disseminate the study results to their members and wider patient communities.

\section{Ethics and dissemination}

The protocol of this study has been approved by the Institutional Review Board of Chang Gung Memorial Hospital (202001 386A3). The study is registered on ClinicalTrials. gov. All participants will have to sign and date an informed consent form. The findings arising from this study will be disseminated in peer-reviewed journals and academic conferences. The datasets during the current study are available from the corresponding author on reasonable request.

\section{Author affiliations}

${ }^{1}$ Department of Neurology, Chang Gung Memorial Hospital Chiayi Branch, Puzi, Taiwan

${ }^{2}$ College of Medicine, Chang Gung University, Taoyuan, Taiwan

${ }^{3}$ Department of Diagnostic Radiology, Chang Gung Memorial Hospital Chiayi Branch, Puzi, Taiwan

${ }^{4}$ Department of Emergency Medicine, Chang Gung Memorial Hospital Chiayi Branch, Puzi, Taiwan

${ }^{5}$ Department of Radiology, Chang Gung Memorial Hospital Chiayi Branch, Puzi, Taiwan

${ }^{6}$ Department of Neurosurgery, Chang Gung Memorial Hospital Chiayi Branch, Puzi, Taiwan

\section{Twitter Hsu-Huei Weng @lukeweng}

Contributors Y-CH and J-TY conceptualised and designed the initial protocol. JDL, L-CL, H-HW and Y-HT amended the initial protocol. Y-CH drafted the manuscript and received the fund. All authors read and approved of the protocol prior to submission.

Funding This work was supported by Chang Gung Memorial Hospital's research grant (grant number: CORPG6K0161).

Competing interests None declared.

Patient and public involvement Patients and/or the public were involved in the design, or conduct, or reporting, or dissemination plans of this research. Refer to the Methods section for further details.

Patient consent for publication Not applicable.

Provenance and peer review Not commissioned; externally peer reviewed.

Open access This is an open access article distributed in accordance with the Creative Commons Attribution Non Commercial (CC BY-NC 4.0) license, which permits others to distribute, remix, adapt, build upon this work non-commercially, and license their derivative works on different terms, provided the original work is properly cited, appropriate credit is given, any changes made indicated, and the use is non-commercial. See: http://creativecommons.org/licenses/by-nc/4.0/.

\section{ORCID iDs}

Yen-Chu Huang http://orcid.org/0000-0001-7885-6376

Hsu-Huei Weng http://orcid.org/0000-0002-2020-8912

Yuan-Hsiung Tsai http://orcid.org/0000-0003-4906-0365

\section{REFERENCES}

1 Charidimou A, Pantoni L, Love S. The concept of sporadic cerebral small vessel disease: a road map on key definitions and current concepts. Int J Stroke 2016;11:6-18.

2 Kim BJ, Kim JS. Ischemic stroke subtype classification: an Asian viewpoint. J Stroke 2014;16:8-17. 
3 Petrone L, Nannoni S, Del Bene A, et al. Branch atheromatous disease: a clinically meaningful, yet unproven concept. Cerebrovasc Dis 2016;41:87-95.

4 Yamamoto Y, Ohara T, Hamanaka M, et al. Characteristics of intracranial branch atheromatous disease and its association with progressive motor deficits. J Neurol Sci 2011;304:78-82.

$5 \mathrm{Kim}$ JS, Yoon Y. Single subcortical infarction associated with parental arterial disease: important yet neglected sub-type of atherothrombotic stroke. Int J Stroke 2013;8:197-203.

6 Ko Y, Lee S, Chung J-W, et al. MRI-based algorithm for acute ischemic stroke subtype classification. J Stroke 2014;16:161-72.

7 Gao S, Wang YJ, Xu AD, et al. Chinese ischemic stroke subclassification. Front Neurol 2011;2:6.

8 Johnston SC, Easton JD, Farrant M, et al. Clopidogrel and aspirin in acute ischemic stroke and high-risk TIA. N Engl J Med 2018;379:215-25

9 Wang Y, Wang Y, Zhao X, et al. Clopidogrel with aspirin in acute minor stroke or transient ischemic attack. N Engl J Med 2013;369:11-19.

10 Kimura T, Tucker A, Sugimura T, et al. Ultra-Early combination antiplatelet therapy with cilostazol for the prevention of branch atheromatous disease: a multicenter prospective study. Cerebrovasc Dis Extra 2016;6:84-95.

11 Wang $\mathrm{C}, \mathrm{Yi} X$, Zhang B, et al. Clopidogrel plus aspirin prevents early neurologic deterioration and improves 6 -month outcome in patients with acute large artery atherosclerosis stroke. Clin Appl Thromb Hemost 2015;21:453-61.

12 Kim D, Park J-M, Kang K, et al. Dual versus mono antiplatelet therapy in large atherosclerotic stroke. Stroke 2019;50:1184-92.

13 Grundy SM, Stone NJ, Bailey AL, et al. 2018 AHA/ACC/AACVPR/ AAPA/ABC/ACPM/ADA/AGS/APhA/ASPC/NLA/PCNA guideline on the management of blood cholesterol: a report of the American College of Cardiology/American heart association Task force on clinical practice guidelines. Circulation 2019;139:e1082-143.

14 Stone NJ, Robinson JG, Lichtenstein AH. ACC/AHA guideline on the treatment of blood cholesterol to reduce atherosclerotic cardiovascular risk in adults: a report of the American College of Cardiology/American heart association Task force on practice guidelines. Circulation 2013;2014:S1-45.

15 Fang J-X, Wang E-Q, Wang W, et al. The efficacy and safety of high-dose statins in acute phase of ischemic stroke and transient ischemic attack: a systematic review. Intern Emerg Med 2017:12:679-87.

16 Chung J-W, Cha J, Lee MJ, et al. Intensive statin treatment in acute ischaemic stroke patients with intracranial atherosclerosis: a highresolution magnetic resonance imaging study (STAMINA-MRI study). J Neurol Neurosurg Psychiatry 2020;91:204-11.

17 Liu D, Liu J, Cai Y, et al. Is the future of symptomatic intracranial atherosclerotic stenosis management promising? J Neurol Neurosurg Psychiatry 2020;91:122-4.

18 Huang W-Y, Lee M, Sung S-F, et al. Atrial fibrillation trial to evaluate real-world procedures for their utility in helping to lower stroke events: a randomized clinical trial. Int J Stroke 2021;16:300-10.

19 Huang Y-C, Tsai Y-H, Lee J-D, et al. A novel neuroimaging model to predict early neurological deterioration after acute ischemic stroke. Curr Neurovasc Res 2018;15:129-37.

20 Collins R, Reith C, Emberson J, et al. Interpretation of the evidence for the efficacy and safety of statin therapy. Lancet 2016;388:2532-61.

21 Siegler JE, Martin-Schild S. Early neurological deterioration (END) after stroke: the END depends on the definition. Int J Stroke 2011;6:211-2.

22 Mehran R, Rao SV, Bhatt DL, et al. Standardized bleeding definitions for cardiovascular clinical trials: a consensus report from the Bleeding Academic Research Consortium. Circulation 2011:123:2736-47.

23 van Rooij WJ, Sprengers ME, de Gast AN, et al. 3D rotational angiography: the new gold standard in the detection of additional intracranial aneurysms. AJNR Am J Neuroradiol 2008;29:976-9. 